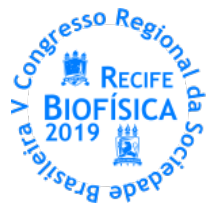

\title{
NOVAS APLICAÇÕES DA CINARIZINA ATRAVÉS DE DRUG DELIVERY COM CUBOSSOMOS, UM FUTURO PROMISSOR
}

\author{
Mayra Lotierzo ${ }^{1 *}$; Bruna Casadei²; Bárbara Malheiros ${ }^{1}$; Raphael D. Castro²; Giovana Firpo²; Leandro R. S. Barbosa ${ }^{2}$ \\ 1'Laboratório de Biossistemas, Departamento de Tecnologia Bioquímica Farmacêutica, Faculdade de Ciências Farmacêuticas, Universidade de São \\ Paulo, São Paulo, SP. \\ ${ }^{2}$ Laboratório de Biossistemas, Departamento de Física Geral, Instituto de Física, Universidade de São Paulo, São Paulo, SP. \\ *may.lotierzo@usp.br
}

\section{RESUMO}

A busca de novas aplicações para medicamentos já aprovados pelas agências de regulação, também chamado de reposicionamento de fármacos, é importante para o tratamento de doenças parasitarias negligenciadas, pois diminui custos e tempo de desenvolvimento farmacêutico. A cinarizina é uma droga já conhecida e estudada para diversas aplicações, atualmente, sua atuação contra leishmaniose vem sendo pesquisada. Devido à estrutura única, os cubossomos possuem a capacidade de incorporar drogas altamente hidrofóbicas, como a cinarizina que possui $\log \mathrm{P}: 5,8$. Cubossomos são nanopartículas que possuem grande potencial para realizar a entrega controlada de fármacos (Drug Delivery), visando melhorar sua eficiência e diminuir possíveis efeitos colaterais. Utilizando as técnicas de espalhamento de raio-x em baixo ângulo (SAXS), espalhamento de luz dinâmico (DLS) e microscopia de transmissão (TEM) estudamos a interferência estrutural da cinarizina em cubossomos de monoleína. A técnica de hemólise foi utilizada para testes iniciais de toxicidade, e HPLC foi realizado para definir a eficiência de encapsulação do fármaco. Para a produção dos cubossomos é utilizado myverol, uma mistura de lipídeos anfifílicos com aproximadamente $60 \%$ de monoleina, e pluronic F127. Os cubossomos de myverol produzidos apresentam estrutura cubica cristalográfica $1 \mathrm{~m} 3 \mathrm{~m}$ com parâmetro de rede a 11 nm e tamanho de $\sim 270 \mathrm{~nm}$. Conseguimos uma eficiência de encapsulação próxima a 100\%. Apoio financeiro: Conselho Nacional de Desenvolvimento Científico e Tecnológico (CNPq); Fundação de Amparo à Pesquisa do Estado de São Paulo (FAPESP). 